\title{
Towards the nucleon hadronic tensor from lattice QCD
}

\author{
Jian Liang $\odot,{ }^{1, *}$ Terrence Draper, ${ }^{1}$ Keh-Fei Liu, ${ }^{1, \dagger}$ Alexander Rothkopf, ${ }^{2}$ and Yi-Bo Yang ${ }^{3}$ \\ $(\chi \mathrm{QCD}$ Collaboration) \\ ${ }^{1}$ Department of Physics and Astronomy, University of Kentucky, Lexington, Kentucky 40506, USA \\ ${ }^{2}$ Faculty of Science and Technology, University of Stavanger, 4021 Stanvanger, Norway \\ ${ }^{3}$ CAS Key Laboratory of Theoretical Physics, Institute of Theoretical Physics, \\ Chinese Academy of Sciences, Beijing 100190, China
}

(Received 5 August 2019; accepted 7 May 2020; published 15 June 2020)

\begin{abstract}
We present the first calculation of the hadronic tensor on the lattice for the nucleon. The hadronic tensor can be used to extract structure functions in deep inelastic scatterings and to provide information for neutrino-nucleon scattering which is crucial to neutrino-nucleus scattering experiments at low energies. The most challenging part in the calculation is to solve an inverse problem. We have implemented and tested three algorithms using mock data, showing that the Bayesian reconstruction method has the best resolution in extracting peak structures while the Backus-Gilbert and maximum entropy methods are somewhat more stable for smooth functions. Numerical results are presented for both the elastic case (clover fermions on domain wall configurations with $m_{\pi} \sim 370 \mathrm{MeV}$ and $a \sim 0.06 \mathrm{fm}$ ) and a case (anisotropic clover lattice with $m_{\pi} \sim 380 \mathrm{MeV}$ and $a_{t} \sim 0.035 \mathrm{fm}$ ) with large momentum transfer. For the former case, the reconstructed Minkowski hadronic tensor gives precisely the vector charge which proves the feasibility of the approach. For the latter case, the resonance and possibly shallow inelastic scattering contributions around energy transfer $\nu=1 \mathrm{GeV}$ are clearly observed but no information is obtained for higher excited states with $\nu>2 \mathrm{GeV}$. A check of the effective masses of the $\rho$ meson with different lattice setups indicates that, in order to reach higher energy transfers, using lattices with smaller lattice spacings is essential.
\end{abstract}

DOI: $10.1103 /$ PhysRevD.101.114503

\section{INTRODUCTION}

In scattering processes involving nucleons such as deep inelastic scattering (DIS) and neutrino-nucleon scattering at low energies, the hadronic tensor $W_{\mu \nu}$ is used to characterize the nonperturbative nature of the nucleon structure. It is the imaginary part of the forward virtual Compton scattering amplitude $W_{\mu \nu}=\frac{1}{2 \pi} \operatorname{Im} T_{\mu \nu}$ and can be expressed as a nucleon matrix element with two current operators inserted,

$$
W_{\mu \nu}=\frac{1}{4 \pi} \int d^{4} z e^{i q \cdot z}\left\langle p, s\left|\left[J_{\mu}^{\dagger}(z) J_{\nu}(0)\right]\right| p, s\right\rangle .
$$

The hadronic tensor of the vector currents can be further decomposed, according to its Lorentz structure, into structure functions, i.e.,

\footnotetext{
jian.liang@uky.edu

tliu@g.uky.edu
}

Published by the American Physical Society under the terms of the Creative Commons Attribution 4.0 International license. Further distribution of this work must maintain attribution to the author(s) and the published article's title, journal citation, and DOI. Funded by SCOAP ${ }^{3}$.
$W_{\mu \nu}=\left(-g_{\mu \nu}+\frac{q_{\mu} q_{\nu}}{q^{2}}\right) F_{1}\left(x, Q^{2}\right)+\frac{\hat{p}_{\mu} \hat{p}_{\nu}}{p \cdot q} F_{2}\left(x, Q^{2}\right)$

for the unpolarized case, where $\hat{p}_{\mu}=p_{\mu}-\frac{p \cdot q}{q^{2}} q_{\mu} \cdot p_{\mu}$ and $q_{\mu}$ are the nucleon 4-momentum and momentum transfer, respectively. $x$ is the Bjorken $x=\frac{Q^{2}}{2 p \cdot q}$. The structure functions are valuable quantities which reveal the inner structure of the nucleon. They can be used to extract parton distribution functions (PDFs) through the QCD factorization theorem $F_{i}=\sum_{a} c_{i}^{a} \otimes f_{a}$, where the convolution kernel $c_{i}^{a}$ is perturbatively calculable.

Due to their nonperturbative nature and importance, it is natural to explore the possibility of calculating the hadronic tensor and structure functions with lattice QCD, which is a first-principles nonperturbative method of solving the strong interaction. While the proposal for a lattice QCDbased evaluation was put forward more than 20 years ago $[1,2]$, it has only recently become feasible to compute the necessary four-point correlation functions, thanks to increases in computing power $[3,4]$. In recent years, there has been a lot of effort in the lattice community focusing on the computation of $x$-dependent PDFs. Examples are quasi-PDFs and LaMET [5,6], Compton amplitude [7], 
pseudo-PDFs [8,9], and lattice cross-sections [10,11]. Each of the approaches has its own advantages and difficulties. For example, some of the approaches try to connect the lattice matrix elements with the light cone PDFs by first combining the matrix elements with different nucleon momentum $\vec{p}$ and spacial distance $\vec{\xi}$ into an Ioffe time $(\vec{p} \cdot \vec{\xi})$ distribution and then using a Fourier transform on the Ioffe time to get the $x$-dependent PDF. So, large $\vec{p}$ and therefore large $\vec{p} \cdot \vec{\xi}$ are essential from them especially to access small $x$. But it is hard to have a highly boosted nucleon on the lattice. In our approach, the hadronic tensor and structure functions account for the general leptonnucleon scattering cross-section. To access the PDFs in the DIS case, what we need is to have the structure functions with large momentum transfer $Q^{2}$ and energy transfer $\nu$. There is no direct requirement of large nucleon momentum $\vec{p}$ since the structure functions in the hadronic tensor are frame independent (N.B. many DIS experiments are done with the nucleon at rest), although we find that a slightly boosted nucleon makes it easier in practice to reach large $\nu$ on the lattice. Another advantage of our method is that the hadronic tensor is scale independent, such that no renormalization is needed except for the finite lattice normalization of the vector current if local operators are used. However, to convert the hadronic tensor from Euclidean space to Minkowski space involves an inverse problem [3] which presents a substantial numerical challenge. In case that reliable lattice results for the Minkowski hadronic tensor are obtained for a set of kinematic setups, they can be used to obtain parton distribution functions via the factorization theorem, as are carried out in global fittings of experiments.

Another feature of calculating the hadronic tensor on the lattice is that it reveals explicitly the connected-sea antiparton contribution. It has been pointed out $[1,12]$ that the Gottfried sum rule violation (i.e., the $\bar{u}$ and $\bar{d}$ difference of their PDFs) can be explained by the existence of the connected-sea antipartons. Recently, the momentum fraction ratio of strange to $u / d$ quark in disconnected insertions was calculated [13], which helps to separate the connectedsea and disconnected-sea parton distributions in global fittings. The calculation of hadronic tensor using Euclidean four-point correlation functions will provide a direct proof to the degrees of freedom of connected-sea antipartons and can finally resolve the puzzle of Gottfried sum rule violation.

In addition to deep inelastic scattering, the hadronic tensor plays an important role too for scatterings at lower energies such as in the experiments of neutrino-nucleus scattering (e.g., the LBNF/DUNE [14] at Fermilab), which aim to study neutrino properties. These experiments face several challenges such as the reconstruction of the neutrino beam energy and flux and the consideration of nuclear effects and models [15]. In view of this, the input of accurate determination of neutrino-nucleon scattering is vital to investigating the nuclear effects of neutrino-nucleus scattering. However, it is not trivial to study the neutrinonucleon scattering since at different beam energies, different contributions [elastic (EL), resonance (RES), shallow inelastic scattering (SIS), and DIS] dominate the total cross-section [16]. Nevertheless, the hadronic tensor is useful in all the energy regions. For example, in the EL region of neutrino-nucleon scattering that is relevant to the quasielastic neutrino-nucleus scattering, the hadronic tensor is actually the square of the elastic form factors of the nucleon and, as a result, the cross-section of the neutrinonucleus scattering can be calculated by combining the nucleon form factors and nuclear models about the nucleon distribution inside a nucleus. In the RES, SIS, and DIS regions, inelastic neutrino-nucleon scatterings emerge and one will need to have the hadronic tensor to cover all the inclusive contributions. In this sense, calculating the hadronic tensor is so far the only way we know that lattice QCD can serve the neutrino experiments in the whole energy range [17]. ${ }^{1}$

Since lattice QCD is formulated with Euclidean time and the hadronic tensor involves a four-dimensional Fourier transform, one cannot calculate the hadronic tensor directly on the lattice. Instead, we calculate its counterpart in Euclidean space and then convert it back to Minkowski space. The formalism of Euclidean hadronic tensor is discussed in Sec. II. The conversion to Minkowski space is implemented by solving the inverse problem which is the most challenging part of our calculation. We will discuss three methods and two examples in Sec. III. Section IV presents numerical results for both the elastic case and a case with large momentum transfer. A discussion on the results comes in Sec. V.

\section{LATTICE FORMALISM OF HADRONIC TENSOR}

After inserting a complete set of intermediate states in Eq. (1) and carrying out the integral, one comes to the following expression of the hadronic tensor [3]:

$$
\begin{aligned}
W_{\mu \nu}^{M}= & \frac{1}{2} \sum_{n} \int \prod_{i}^{N_{n}}\left[\frac{d^{3} \vec{p}_{i}}{(2 \pi)^{3} 2 E_{i}}\right]\left\langle p, s\left|J_{\mu}^{\dagger}(0)\right| n\right\rangle\langle n| J_{\nu}(0) \\
& \times|p, s\rangle(2 \pi)^{3} \delta^{4}\left(q-p_{n}+p\right),
\end{aligned}
$$

where $q$ is the momentum transfer, $p$ the nucleon momentum, and $p_{n}$ the momentum of the $n$th intermediate state. $\prod_{i}^{N_{n}}\left[\frac{d^{3} \vec{p}_{i}}{(2 \pi)^{3} 2 E_{i}}\right]$ gives the normalization of the intermediate states, and the index $i$ goes from 1 to $N_{n}$, which is the number of hadrons contained in the state $n$. The fourdimensional Dirac delta function ensures the conservation of 4-momentum and picks out the contribution of a

\footnotetext{
${ }^{1}$ We thank A. Kronfeld for bringing to our attention the relevance of the hadronic tensor to the neutrino-nucleon scattering.
} 
particular momentum transfer. However, it is noted in [3] that if one tries to carry out the integral of Eq. (1) in the Euclidean case, the Fourier transform in the time direction becomes a Laplace transform,

$$
\begin{aligned}
W_{\mu \nu}^{\prime}= & \frac{1}{4 \pi} \sum_{n} \int d t e^{\left(\nu-\left(E_{n}-E_{p}\right)\right) t} \\
& \times \int d^{3} \vec{z} e^{i \vec{q} \cdot \vec{z}}\left\langle p, s\left|J_{\mu}^{\dagger}(\vec{z})\right| n\right\rangle\left\langle n\left|J_{\nu}(0)\right| p, s\right\rangle,
\end{aligned}
$$

and after the integration one has

$$
\begin{aligned}
W_{\mu \nu}^{\prime}= & \frac{1}{4 \pi} \sum_{n} \frac{e^{\left(\nu-\left(E_{n}-E_{p}\right)\right) T}-1}{\nu-\left(E_{n}-E_{p}\right)} \\
& \times \int d^{3} \vec{z} e^{i \vec{q} \cdot \vec{z}}\left\langle p, s\left|J_{\mu}^{\dagger}(\vec{z})\right| n\right\rangle\left\langle n\left|J_{\nu}(0)\right| p, s\right\rangle,
\end{aligned}
$$

where $\nu$ is the energy transfer, $E_{p}$ and $E_{n}$ are the energies of the external nucleon and the $n$th intermediate state, and $T$ is the integration length in the time direction. The factor of $\frac{e^{\left(\nu-\left(E_{n}-E_{p}\right)\right) T}-1}{\nu-\left(E_{n}-E_{p}\right)}$ is problematic since it does not converge if $\nu-\left(E_{n}-E_{p}\right)>0$. This happens when $\nu$ is greater than the energy gap between the nucleon and the intermediate states, such as $\Delta$, Roper, ... . Besides, even if the numerator converges, $\frac{1}{\nu-\left(E_{n}-E_{p}\right)}$ is not a good approximation to the delta function and one cannot pick out the clean contribution for a specific $\nu$ as nearby states will mix.

Instead, we construct the following four-point correlation function with only a three-dimensional Fourier transform:

$$
\begin{aligned}
C_{4}\left(t_{f}, t_{2}, t_{1}\right)= & \sum_{\vec{x}_{f}} e^{-i \vec{p} \cdot \vec{x}_{f}} \sum_{\vec{x}_{1} \vec{x}_{2}} e^{-i \vec{q} \cdot\left(\vec{x}_{2}-\vec{x}_{1}\right)} \\
& \times\left\langle\chi_{N}\left(\vec{x}_{f}, t_{f}\right) J_{\mu}^{\dagger}\left(\vec{x}_{2}, t_{2}\right) J_{\nu}\left(\vec{x}_{1}, t_{1}\right) \bar{\chi}_{N}\left(\overrightarrow{0}, t_{0}\right)\right\rangle
\end{aligned}
$$

and also the nucleon two-point function as

$$
C_{2}\left(t_{f}\right)=\sum_{\vec{x}_{f}} e^{-i \vec{p} \cdot \vec{x}_{f}}\left\langle\chi_{N}\left(\vec{x}_{f}, t_{f}\right) \bar{\chi}_{N}\left(\overrightarrow{0}, t_{0}\right)\right\rangle,
$$

where $\chi_{N}(\vec{x}, t)$ is the nucleon interpolation field operator. Then, the Euclidean hadronic tensor $W_{\mu \nu}^{E}(\vec{p}, \vec{q}, \tau)$ is defined by the ratio of the four-point function to the two-point function $[1-4,12]$ as

$$
\begin{aligned}
W_{\mu \nu}^{E}(\vec{p}, \vec{q}, \tau)= & \frac{E_{p}}{m_{p}} \frac{\operatorname{Tr}\left[\Gamma_{e} C_{4}\right]}{\operatorname{Tr}\left[\Gamma_{e} C_{2}\right]} \\
\stackrel{t_{f} \gg t_{2}, t_{1} \gg t_{0}}{\longrightarrow} & \sum_{\vec{x}_{1} \vec{x}_{2}} e^{-i \vec{q} \cdot\left(\vec{x}_{2}-\vec{x}_{1}\right)} \\
& \times\left\langle p, s\left|J_{\mu}^{\dagger}\left(\vec{x}_{2}, t_{2}\right) J_{\nu}\left(\vec{x}_{1}, t_{1}\right)\right| p, s\right\rangle e^{E_{p} \tau},
\end{aligned}
$$

where $E_{p}$ and $m_{p}$ are the energy and mass of the nucleon, and $\Gamma_{e}=\frac{1+\gamma_{4}}{2}$ is the unpolarized projector and $\tau=t_{2}-t_{1}$. We can insert the intermediate states again between the two currents and we have

$$
W_{\mu \nu}^{E}=\sum_{n} A_{n} e^{-\left(E_{n}-E_{p}\right) \tau},
$$

where

$$
A_{n} \equiv \sum_{\vec{x}_{1} \vec{x}_{2}} e^{-i \vec{q} \cdot\left(\vec{x}_{2}-\vec{x}_{1}\right)}\left\langle p, s\left|J_{\mu}^{\dagger}\left(\vec{x}_{2}, 0\right)\right| n\right\rangle\left\langle n\left|J_{\nu}\left(\vec{x}_{1}, 0\right)\right| p, s\right\rangle .
$$

The computation of four-point functions consumes the most computer resources in our calculation. Doing the Wick contraction for the four-point function leads to several topologically distinct diagrams in the Euclidean path-integral formulation (shown in Fig. 1). We have not specified the flavor of the quark lines in the figure. In practice where the flavor is taken into account, the contractions can be more complicated according to the types of the currents (neutral or charged). For the calculation with small momentum transfers, all the diagrams contribute and one needs to combine them to have physical results. However, for the case with large momentum and energy transfers as in the DIS, Figs. 1(a)-1(c) are dominated by the leading twist while Figs. 1(d)-1(f) are suppressed since they involve only high twists. Therefore, the respective leading twist parton degrees of freedom are classified by the first three diagrams, namely, the valence and connectedsea (CS) partons $q^{\mathrm{v}+\mathrm{cs}}$ [Fig. 1(a)], the CS antipartons $\bar{q}^{\mathrm{cs}}$ [Fig. 1(b)], and the disconnected-sea (DS) partons and antipartons $q^{\mathrm{ds}}+\bar{q}^{\mathrm{ds}}$ [Fig. 1(e)] [1,2]. It is a great feature of our approach that they all can be calculated separately, especially since the CS antipartons are responsible for the Gottfried sum rule violation [1,2,12].

After the Euclidean hadronic tensor is calculated, we need to convert it back to Minkowski space to obtain physical results. Formally, the inverse Laplace transform fulfills this objective,

$$
W_{\mu \nu}^{M}(\vec{p}, \vec{q}, \nu)=\frac{1}{i} \int_{c-i \infty}^{c+i \infty} d \tau e^{\nu \tau} W_{\mu \nu}^{E}(\vec{p}, \vec{q}, \tau) .
$$

However, in practice, the Euclidean hadronic tensor is a function of Euclidean time, which is real, so that the integral in the inverse Laplace transform along the imaginary time axis is not possible. Numerically, one can try to solve the inverse problem of the Laplace transform to obtain an estimation of $W_{\mu \nu}^{M}[3,4]$,

$$
W_{\mu \nu}^{E}(\vec{p}, \vec{q}, \tau)=\int d \nu W_{\mu \nu}^{M}(\vec{p}, \vec{q}, \nu) e^{-\nu \tau}
$$




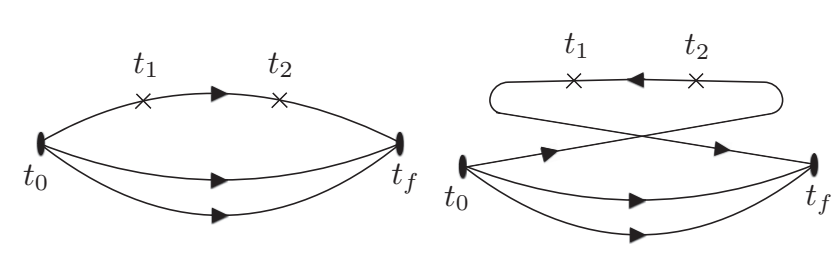

(a)

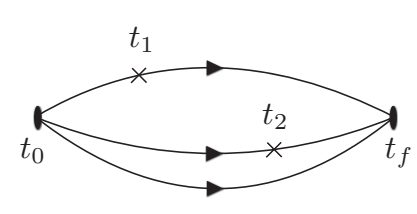

(d) (b)

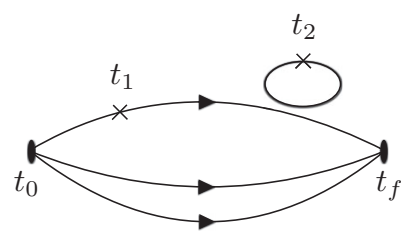

(e)

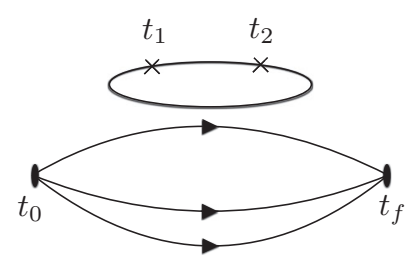

(c)

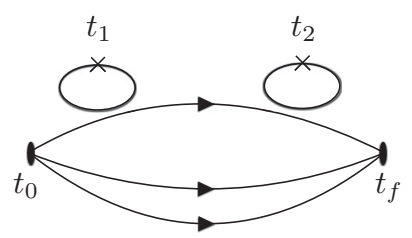

(f)

FIG. 1. Topologically distinct diagrams in the Euclidean path-integral formulation of the nucleon hadronic tensor. (a)-(c) contain all twists and (d)-(f) contain high twists only.

Details about solving the inverse problem are discussed in Sec. III.

\section{SOLVING THE INVERSE PROBLEM}

A general form of the inverse problem reads

$$
c\left(\tau_{i}\right)=\int k\left(\tau_{i}, \nu\right) \omega(\nu) d \nu
$$

where $c\left(\tau_{i}\right)$ denotes discrete lattice data with a finite number of points [usually $O(10)$ ], $k\left(\tau_{i}, \nu\right)$ is the integral kernel that is a function of both $\tau_{i}$ and $\nu$, and $\omega(\nu)$ is the target function which is usually continuous with respect to $\nu$. In principle, determining every detail of a totally unknown continuous function with finite input information is not possible; videlicet, more than one solution can be found to match the input data. Numerically, we can always discretize $\omega(\nu)$,

$$
c\left(\tau_{i}\right)=\sum_{j} k\left(\tau_{i}, \nu_{j}\right) \omega\left(\nu_{j}\right) \Delta \nu_{j},
$$

however, the number of $\nu_{j}$ one needs to reproduce the structures of $\omega(\nu)$ is, in many cases, much larger than the number of input points, so the problem is still ill-posed. Nevertheless, many algorithms are available to extract the most probable solution of $\omega$ at a certain resolution. Actually, this is a common problem not only in physics, and the algorithms have been kept updated and improved.

In this section, we will briefly introduce three methods to solve the inverse problem, discuss their features, and use some mock data to test their resolutions and robustness.

\section{A. Backus-Gilbert method}

The Backus-Gilbert (BG) method [18-20] utilizes the fact that the kernel functions, if they span a complete function basis, can be linearly combined to approximate a delta function,

$$
\sum_{i} a\left(\tau_{j}, \nu_{0}\right) k\left(\tau_{i}, \nu\right) \sim \delta\left(\nu-\nu_{0}\right),
$$

where $a\left(\tau_{i}, \nu_{0}\right)$ are the coefficients for the $i$ th kernel function at a certain point $\nu_{0}$, which can be calculated by assuming a criterion of "deltaness" and solving linear equations. Having $a\left(\tau_{i}, \nu_{0}\right)$, the value of the target function at $\nu_{0}$ can be accessed by

$$
\sum_{i} a\left(\tau_{i}, \nu_{0}\right) c\left(\tau_{i}\right) \sim \int \delta\left(\nu-\nu_{0}\right) \omega(\nu) d \nu=\omega\left(\nu_{0}\right) .
$$

It is worthwhile noting that the number of independent kernel functions is equal to the number of the discrete lattice data points so usually the function basis spanned by the kernel functions is far from complete, leading to a coarse resolution. In some sense, this is a feature instead of a disadvantage because the lattice spectrum is discrete and the volume correction for a particular multiparticle state is not negligible, but the broadened delta function ("regulated delta function") can be treated as a smoothing procedure and ensures a well-defined infinite volume limit [20]. However, in our case, we are only concerned about the inclusive contribution rather than the contribution from one particular state, so the infinite volume limit is well-defined. And in fact, it is nearly impossible to isolate the multiparticle-state contributions even with other methods of 
better resolution from present-day lattice data. Another feature of BG is that, unlike other Bayesian-type methods, it solves the problem point by point and does not guarantee that the reconstructed result reproduces the input data well [21], so careful checks are always needed.

\section{B. Maximum entropy method}

The maximum entropy (ME) method [22,23] makes use of Bayesian probability with prior information about the target function to find the most probable solution,

$$
P[\omega \mid D, \alpha, m] \propto \frac{1}{Z_{S} Z_{L}} e^{Q(\omega)},
$$

where $P[\omega \mid D, \alpha, m]$ denotes the conditional probability that $\omega$ is the solution given lattice data $D$, prior information $m$, and a hyper parameter $\alpha . Q=\alpha S-L$ is a combination of the Shannon entropy,

$S=\sum_{j}\left[\omega\left(\nu_{j}\right)-m\left(\nu_{j}\right)-\omega\left(\nu_{j}\right) \log \left(\frac{\omega\left(\nu_{j}\right)}{m\left(\nu_{j}\right)}\right)\right] \Delta \nu_{j}$,

which entails the constraint from the prior information, and the likelihood function

$$
L=\frac{1}{2} \sum_{i, j}\left(c\left(\tau_{i}\right)-c^{\omega}\left(\tau_{i}\right)\right) C_{i j}^{-1}\left(c\left(\tau_{j}\right)-c^{\omega}\left(\tau_{j}\right)\right),
$$

which embodies the constraint from the data. In the equations, $m\left(\nu_{j}\right)$ is the default model (the prior information we plug in), $c\left(\tau_{i}\right)$ is the input Euclidean correlator, $c^{\omega}\left(\tau_{i}\right)$ is the correlator regenerated from $\omega$ using Eq. (14), $C$ is the covariance matrix, and $Z_{S} Z_{L}$ is the normalization factor. $\alpha$ is the weight that balances the two constraints. If $\alpha$ is zero, ME reduces to the normal $\chi^{2}$ fit which has no unique solution for the inverse problem, since the number of parameters is larger than the number of input data. The uniqueness is guaranteed for a finite $\alpha$ and the results of different $\alpha$ 's are averaged in a range of $\alpha$ based on certain assumptions [23]. Practically, the parameter space for finding the maximum probability $P[\omega \mid D, \alpha, m]$, i.e., the maximum value of $Q$, is reduced to a smaller one instead of the whole $\nu$ space by employing singular value decomposition $[23,24]$, which makes the maximum search easier but the resolution may be affected. Improved ME with an extended search space [25] is also proposed and we will check whether it produces better results in our future study.

\section{Bayesian reconstruction}

Bayesian reconstruction (BR) [26] is an improved Bayesian method. The Bayesian probability is

$$
P[\omega \mid D, \alpha, m] \propto e^{Q^{\prime}(\omega)},
$$

where $Q^{\prime}=\alpha S^{\prime}-L-\gamma\left(L-N_{\tau}\right)^{2}$ and

$$
S^{\prime}=\sum_{j}\left[1-\frac{\omega\left(\nu_{j}\right)}{m\left(\nu_{j}\right)}+\log \left(\frac{\omega\left(\nu_{j}\right)}{m\left(\nu_{j}\right)}\right)\right] \Delta \nu_{j},
$$

which is an alternative way to encode the constraint from the prior. $\gamma$ is a numerically large number such that the term $\gamma\left(L-N_{\tau}\right)^{2}$ helps to prevent overfitting. The hyper parameter $\alpha$ here is explicitly integrated over as

$$
P[\omega \mid D, m]=\frac{P[D \mid \omega, I]}{P[D \mid m]} \int d \alpha P[\alpha \mid D, m],
$$

where $P[\alpha \mid D, m]$ is the probability of $\alpha$ in the presence of $D$ and $m$. The search space of the maximum of BR is the whole parameter space which enhances the ability of finding the real maximum while the price to pay is the need of high-precision architecture (e.g., 512-bit floating point numbers). For both Bayesian-type methods, ME and $\mathrm{BR}$, the choice of the default model is in principle arbitrary, but a reliable reconstruction should not depend on the default model. In all the following calculations of the paper, the default model is chosen to be a constant.

\section{Mock data examples}

To test the three methods, mock data are generated. The first set of mock data is a two-point function with three states of mass 1.0, 1.5, and $1.8 \mathrm{GeV}$, respectively, and the spectral weights are all unity. The lattice spacing is tuned to $0.1 \mathrm{fm}$ and the number of time slices available is 20 . Noises are added by assuming normal distributions around the central values and the signal-to-noise ratio is set to be 100 . This is to check the resolution for peak structures of the spectral functions.

The results of the reconstruction can be found in Fig. 2 . The left panel shows the reconstructed spectral functions, and the right panel shows the comparison between the original input data and the two-point functions computed using the resultant spectral functions. For the spectral functions, we see that the result of BG shows basically no peak structures, reflecting its poor resolution for this mock data setup. The result of ME is much better as two peaks around 1 and $2 \mathrm{GeV}$ are clearly seen. Considering the broad widths of the peaks, it is consistent with the input data. BR gives the best reconstruction in this case as two sharp peaks appear at $\sim 1$ and $\sim 1.6 \mathrm{GeV}$. Although the second and the third states are still not separated, the resolution of BR is much better. From the right panel, we see that the regenerated two-point functions from the spectral functions of ME and BR are well consistent with the input data, but for the BG case, differences occur at large $t$. This exhibits the fact that, as pointed out before, BG does not guarantee that the reconstructed result reproduces the input data. 

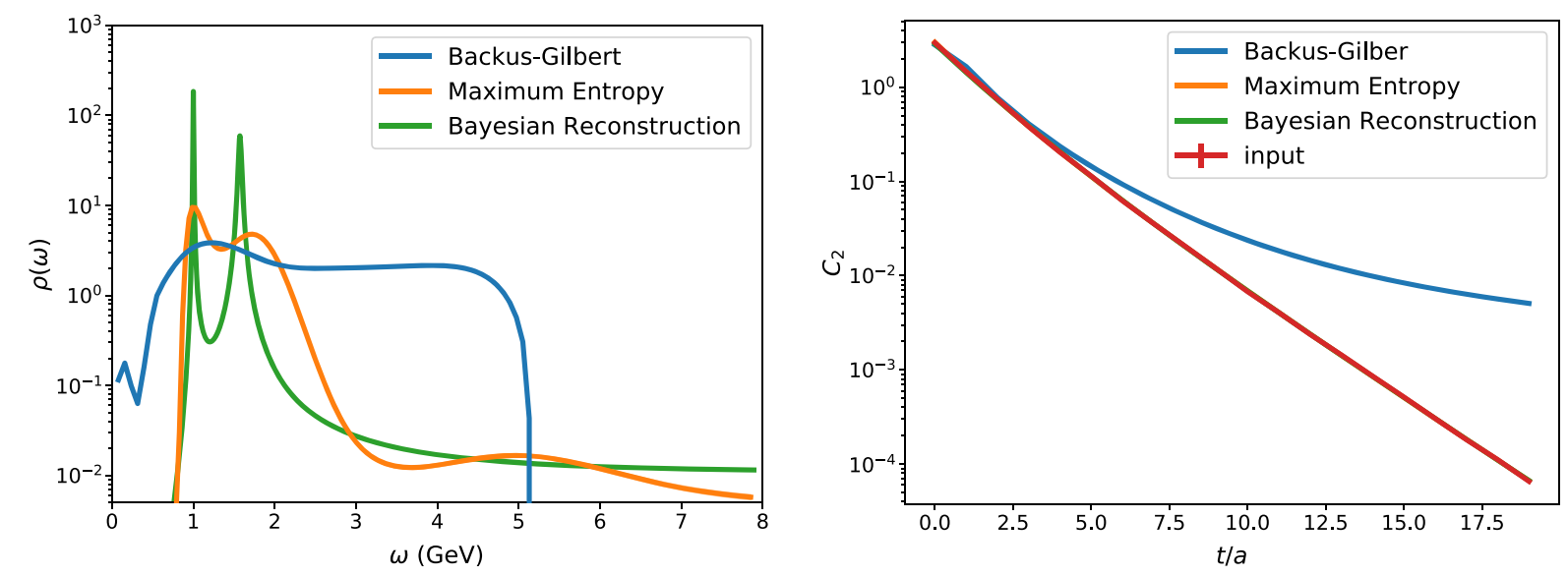

FIG. 2. The results of the reconstruction of the first mock data set. The left panel shows the spectral functions, and the right panel shows the comparison between the original input data and the two-point functions regenerated using the resultant spectral functions. Note that both the ME and BR results coincide with the input data so the orange and green curves are not visible in the right panel. BR has the best resolution in the test.

The second set of the mock data is for the Euclidean hadronic tensor $W^{E}$ with two isolated states of mass 1.0 and $1.5 \mathrm{GeV}$ (simulating the elastic and resonance contributions) and a dense spectrum from 2 to $15 \mathrm{GeV}$ (simulating the contributions of continuous spectrum in SIS and DIS regions). The corresponding matrix elements of these dense states are all assumed to be a constant. This is like the energy dependence of the neutrino-nucleon scattering cross-section. The lattice spacing is set to be $0.02 \mathrm{fm}$ in this case and the number of time slices is 100 . The signalto-noise ratio is set to be 100 too. This example is mainly to check the three methods' ability of resolving a smooth Minkowski hadronic tensor $W^{M}$ at higher energies. The results of this test are shown in Fig. 3. Similarly, the left panel shows the reconstructed Minkowski hadronic tensor and the right panel shows the comparison between the original input data and the Euclidean hadronic tensor recomputed using the resultant Minkowski hadronic tensor.

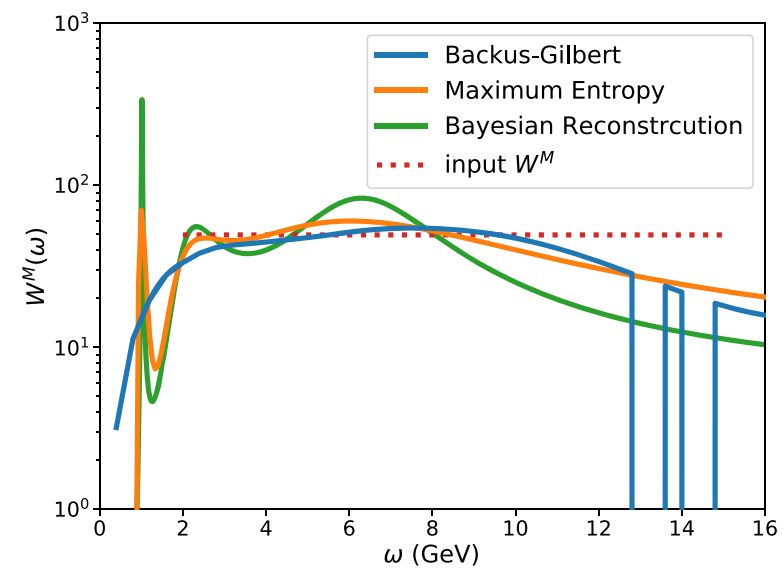

From the left panel, we see that both ME and BR reconstruct a peak at $\sim 1 \mathrm{GeV}$ (the $\mathrm{BR}$ one is much sharper) while BG shows no peak in that low energy region. This agrees with what we observed in the previous test. However, in the region 2-8 GeV, BG presents more consistent results with the input $W^{E}$ (the red dashed line) while both ME and BR show unphysical oscillations. These unphysical oscillations are called "ringing" and are artifacts of the reconstruction [21]. The ringing of the ME method seems weaker, which is due to the fact that the singular value decomposition (SVD) used in ME acts as an additional smoothing that suppresses the ringing but also leads to the significantly larger width of peak structures. Both the BR and ME methods suffer from this disadvantage, so they are not the optimal method for the reconstruction of a smooth hadronic tensor at high energies. Actually, there is an update of BR aiming to address this problem $[21,27]$ and we will also try to test this in our future study. Similar to the

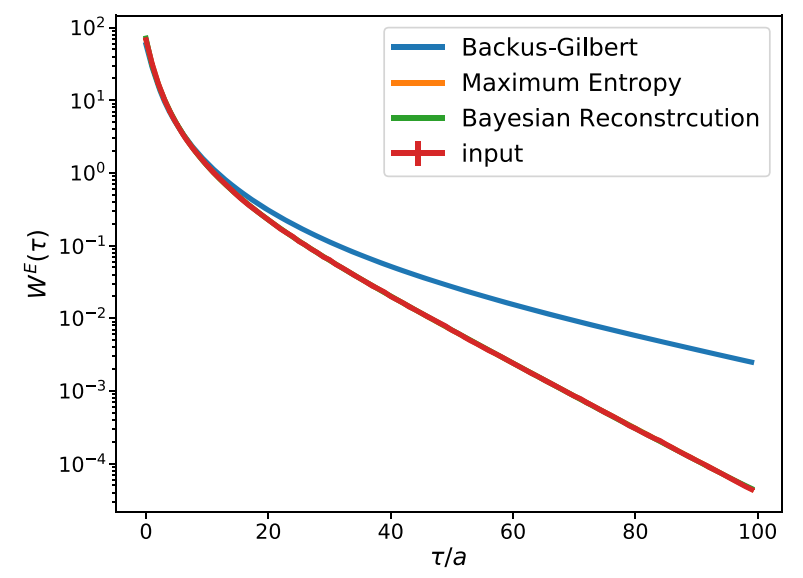

FIG. 3. The test results for the second mock data set. Again, both the ME and BR results coincide with the input data so the orange and green curves are not visible in the right panel. 
first case, the right panel of Fig. 3 shows that the regenerated Euclidean hadronic tensor from ME and BR is well consistent while that of BG is not.

Solving the inverse problem is the most challenging part of our calculation. More detailed studies on how the lattice spacing, the error of the correlation functions, and the number of available time slices affect the reconstruction and more inverse methods regarding this physics problem are needed. Recently, several inverse algorithms have been checked for the efficiency of obtaining $x$-dependent PDFs from mock Euclidean correlators [28]. From the above two tests, we know that BR has the best resolution for peak structures while BG and ME are more stable for resolving smooth functions. Knowing the different methods' advantages, one can combine them to resolve different parts of the Minkowski hadronic tensor, e.g., BR for the sharp peak structures and ME for the smooth region.

\section{RESULTS}

\section{A. Elastic case}

Having discussed how to solve the inverse problem, we now apply the algorithms to realistic lattice data. The first example is to check the vector charge. This is a calculation that is relevant to the neutrino-nucleon scattering and also serves as a benchmark of the whole approach. The calculation is done on RBC/UKQCD domain wall lattice 32If [29] with clover fermions as valence quarks. The configuration is preprocessed by hypercubic (HYP) smearing, and the tadpole improved clover coefficient $C_{\mathrm{sw}}=$ 1.033 is used to generate the clover term for the valence part. The pion mass is tuned to be close to the unitary point $\sim 371 \mathrm{MeV}$. The lattice spacing is about $0.06 \mathrm{fm}$ which we expect to be fine enough such that the inverse algorithms can give reasonably reliable results.

For this case, we choose $\mu=\nu=4$ and $\vec{p}=\vec{q}=0$ to calculate the Euclidean hadronic tensor $W_{\mu \nu}^{E}$. So Eq. (8) becomes
$W_{44}^{E}(\overrightarrow{0}, \overrightarrow{0}, \tau)=\sum_{n}\left\langle p, s\left|\bar{\psi} \gamma_{4} \psi\right| n\right\rangle\left\langle n\left|\bar{\psi} \gamma_{4} \psi\right| p, s\right\rangle e^{-\left(E_{n}-M_{p}\right) \tau}$

For simplicity, the two currents are both inserted on the $d$ quark line, so only Fig. 1(a) contributes. With $\tau \gg 0$ only the ground state survives, so $W_{4,4}^{E}(\overrightarrow{0}, \overrightarrow{0}, \infty)=g_{V}^{2}=1$, given proper normalization factor $Z_{V}$. Two sequential propagators are used for constructing the four-point function with one starting from $t_{0}$ through $t_{1}$ to $t_{2}$ and the other starting from $t_{0}$ through $t_{f}$ to $t_{2}$ [Fig. 1(a)]. Therefore, for one calculation, the source point $t_{0}$ and the two sequential points $t_{1}$ and $t_{f}$ are fixed while all the values of $t_{2}$ are available. In this particular example, we choose $t_{0}=0$, $t_{f}=15$, and $t_{1}=5$ in lattice unit so the working $t_{2}$ should be in the range of $[6,14]$ to exclude the contact points and the corresponding $\tau=t_{2}-t_{1}$ is from 1 to 9 . The result of $W_{44}^{E}(\tau)$ is plotted in Fig. 4(a). It shows that within errors, $W_{4,4}^{E}(\tau)$ is a constant of value 1 , indicating that the excitedstate contributions are negligible. The drop at $\tau=9$ is likely due to the fact that it is too close to the nucleon sink. The errors are around $0.4 \%$ and the number of configurations used is 100 .

To convert the results to Minkowski space, the ME and BG methods are employed. Actually, the exact form of the hadronic tensor for elastic scatterings [30] is

$$
\begin{aligned}
W_{44}^{M}\left(q^{2}, \nu\right)= & \delta\left(q^{2}+2 m_{p} \nu\right) \frac{2 m_{p}}{1-q^{2} / 4 m_{p}^{2}} \\
& \times\left(G_{E}^{2}\left(q^{2}\right)-\frac{q^{2}}{4 m_{p}^{2}} G_{M}^{2}\left(q^{2}\right)\right) .
\end{aligned}
$$

In our case, $W_{44}^{M}\left(q^{2}=0, \nu\right)=\delta(\nu) G_{E}^{2}(0)=\delta(\nu)$. This is easy to understand since the Minkowski hadronic tensor should be a Dirac delta function at $\nu_{0}$ when its Euclidean counterpart is a single exponential $\sim e^{-\nu_{0} t}$ and here the constant Euclidean hadronic tensor is a special case of an exponential with $\nu_{0}=0$.

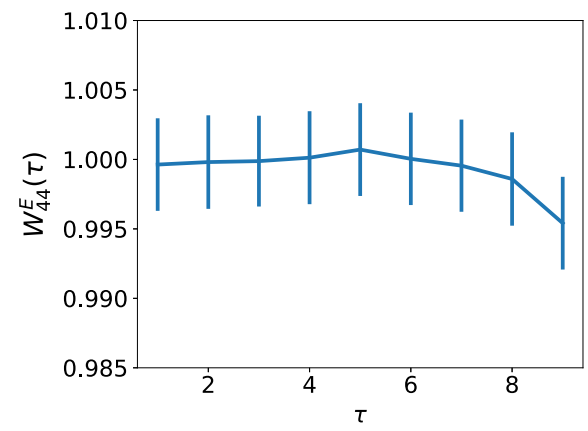

(a)

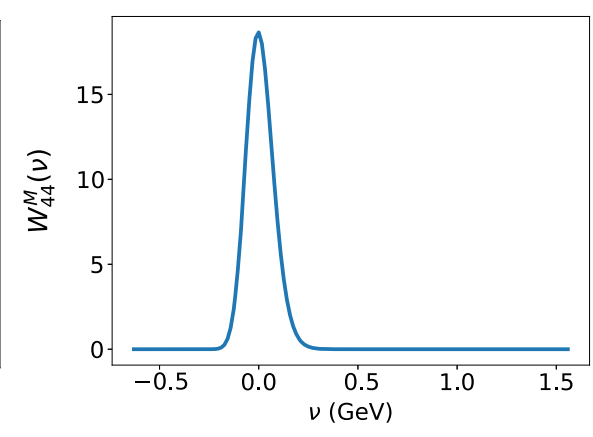

(b)

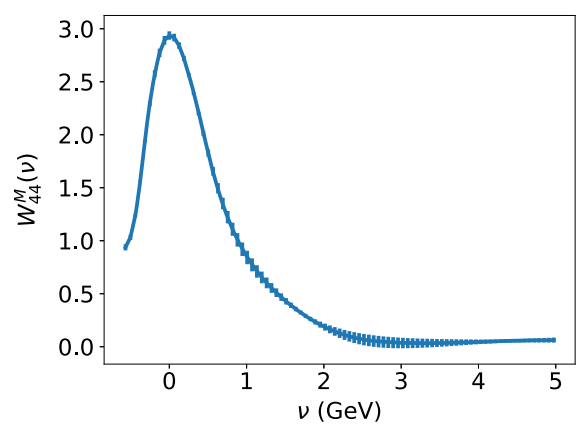

(c)

FIG. 4. The results of the elastic case. (a) The Euclidean hadronic tensor $W_{44}^{E}$ as a function of $\tau$. (b) The Minkowski hadronic tensor $W_{44}^{M}$ as a function of energy transfer $\nu$ from the ME method. (c) The Minkowski hadronic tensor as a function of $\nu$ from the BG method. 
TABLE I. The kinematic setup of nucleon momentum $\vec{p}, 3$-momentum transfer $\vec{q}$, proton energy $E_{p}$, the energy of the lowest intermediate state $E_{n=0}$, the modulus of the 3-momentum transfer $|\vec{q}|$, the range of energy transfer $\nu$, the range of 4-momentum transfer $Q^{2}$, and the corresponding Bjorken $x$.

\begin{tabular}{lccccccc}
\hline \hline$\vec{p}\left(2 \pi / L_{s}\right)$ & $\vec{q}\left(2 \pi / L_{s}\right)$ & $E_{p}(\mathrm{GeV})$ & $E_{n=0}(\mathrm{GeV})$ & $|\vec{q}|(\mathrm{GeV})$ & $\nu(\mathrm{GeV})$ & $Q^{2}\left(\mathrm{GeV}^{2}\right)$ & $x$ \\
\hline \hline$(0,3,3)$ & $(0,-6,-6)$ & 2.15 & 2.15 & 3.57 & {$[2.96,3.68]$} & {$[4,2]$} & {$[0.16,0.07]$} \\
\hline \hline
\end{tabular}

The converted results of $W_{44}^{M}(\nu)$ using ME and BG are plotted in Figs. 4(b) and 4(c), respectively. They both give a peak around $\nu=0$ and in this sense the results are consistent with the theoretical prediction of $\delta(v)$. However, similar to the cases of the mock data, ME shows much better resolution than BG. Another problem of the result of $\mathrm{BG}$ is that it is not symmetric about $\nu=0$, which is because BG has difficulties in resolving the target function with negative $\nu$. An important check is that the area under the peaks should be $g_{V}^{2}=1$, and the values of numerical integral of the results from ME and BG are 1.001(7) and 1.18(6), respectively. Again, ME shows a more precise result.

Although it is cumbersome and unnecessary to calculate the vector charge by constructing four-point functions and solving the inverse problem, it nevertheless shows the feasibility of our approach. The vector charge can be obtained reliably. For more complicated cases such as nonzero momentum transfers or charged currents, this approach can show its advantages and provide the inclusive contribution of all intermediate states.

\section{B. Nonzero nucleon momentum and momentum transfer}

As pointed out in the introduction, another important motivation of calculating the hadronic tensor is to have the lattice results of structure functions in the DIS region which can be used together with experimental inputs to better pin down the parton distribution functions. To this end, we need to have large momentum transfer $Q^{2}$ and energy

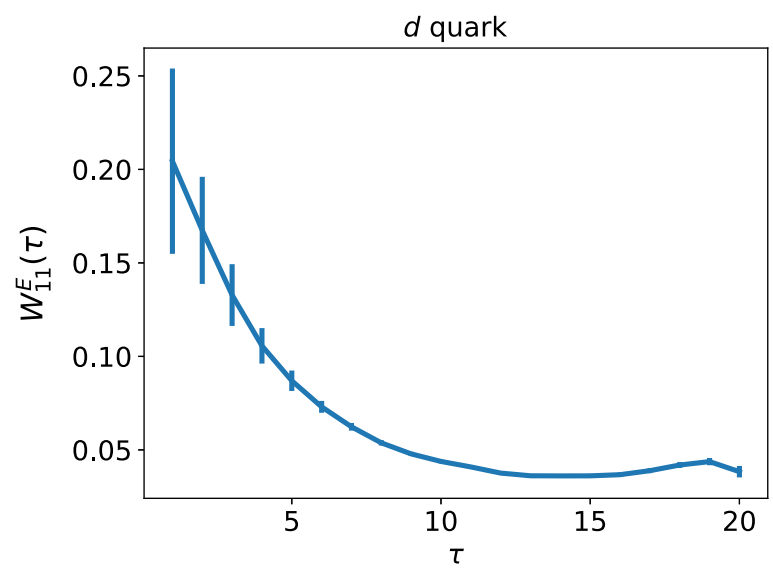

transfer $\nu$ to make the scattering "deep" enough to access the parton degrees of freedom. This is different from the requirement of, e.g., the quasi-PDF approach where they need large nucleon momentum. In practice, in order to reach small $x$ (e.g., $\sim 0.1$ ), it is also beneficial numerically in our approach to have a nonzero 3-momentum of the external proton to be in the opposite direction of the 3-momentum transfer.

For this calculation, we use an anisotropic clover lattice [31] with $a_{t} \sim 0.035 \mathrm{fm}$. The pion mass is about $380 \mathrm{MeV}$ and the momentum unit is $\frac{2 \pi}{L_{s}} \sim 0.42 \mathrm{GeV}$. The reason we switch to this lattice is that the signal-to-noise ratio will be much worse than the previous elastic case when the momentum transfer is large. Thus, having more data points in the $t$ direction helps the inverse algorithms to have more stable results.

The detailed kinetic setup is listed in Table I. We choose $\vec{p}=(0,3,3)$ and $\vec{q}=(0,-6,-6)$ in lattice unit and $\mu=\nu=1$, such that only the $F_{1}$ structure function survives; thus, $W_{11}^{M}=F_{1}\left(x, Q^{2}\right)$. Since the energy transfer $\nu$ is not fixed by the lattice three-dimensional Fourier transform, we can choose a range of $\nu \in[2.96,3.68] \mathrm{GeV}$ such that the corresponding $Q^{2}$ is in a range of $2-4 \mathrm{GeV}^{2}$. The Bjorken $x$ that can be accessed accordingly is between 0.07 and 0.16 for this setup. A special feature of this setup is that $\vec{p}+\vec{q}=-\vec{p}$; therefore, the energy of the lowest intermediate state is $E_{n=0}=E_{p}$ and, for large enough $\tau$, $W_{11}^{E} \propto e^{-\left(E_{n=0}-E_{p}\right) \tau}$ is a constant.

The results of the Euclidean hadronic tensor $W_{11}^{E}(\tau)$ for both $d$ and $u$ quarks are shown in Fig. 5. In this calculation,

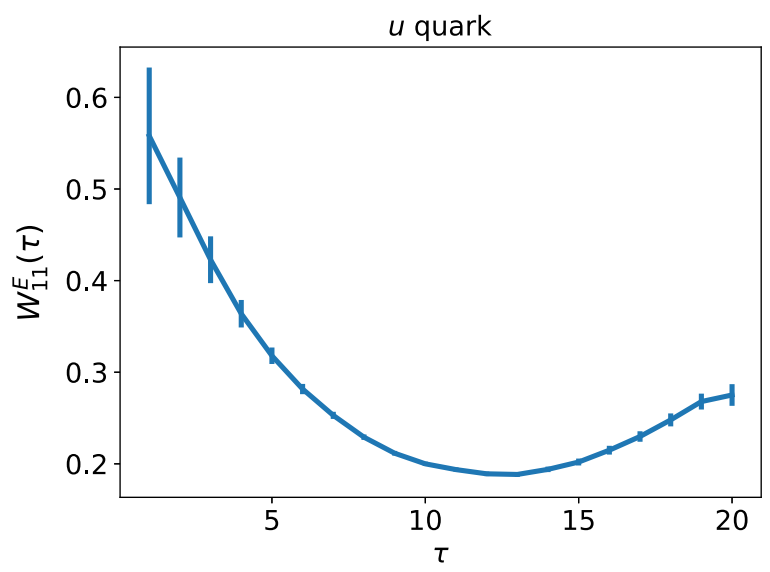

FIG. 5. The Euclidean hadronic tensor $W_{11}^{E}$ as a function of $\tau$ for both $d$ and $u$ quarks. $\vec{p}=(0,3,3)$ and $\vec{q}=(0,-6,-6)$ in lattice unit in this case. 
we set $t_{0}=0, t_{1}=8$, and $t_{f}=28$ in lattice unit and still we focus on the diagram of Fig. 1(a) for the moment. The figures show that for small $\tau$, the Euclidean hadronic tensor $W_{11}^{E}(\tau)$ decays exponentially since the energy of the intermediate states $E_{n}$ is larger than $E_{p}$. For larger $\tau$, it gets flatter for the $d$ quark case (left panel), which is consistent with what we expect, i.e., $E_{n=0}=E_{p}$. The tail of the Euclidean hadronic tensor for the $u$ quark case (right panel) goes up after $\tau \sim 15$, which we believe is due to the contamination of the sink nucleon excited states.

Similarly, we need to solve the inverse problem to obtain the results in Minkowski space. The results from the ME method are shown in Fig. 6. The error bands are mainly from the average of different default models. The behaviors of $d$ and $u$ quarks are similar. We do not observe a peak around the elastic point $\nu=0$ which is because at this point the hadronic tensor is the square of the electric form factor and the form factor is highly suppressed by the large momentum transfer. Taking a dipole form for the form factor with $Q^{2}=12.7 \mathrm{GeV}^{2}$ in this case, the hadronic tensor is suppressed by a factor of $\left(1+Q^{2} / 0.71\right)^{-4} \sim 10^{-5}$ as compared to the charge at $Q^{2}=0$. Then, we do observe a broad structure at about $1 \mathrm{GeV}$, which should account for the combined contribution of nucleon resonances and possibly the SIS.

As discussed above, the preferred $\nu$ range that can lead us to the parton structure functions is from 2.96 to $3.68 \mathrm{GeV}$; however, our results give basically zero within errors in that region. To check whether this is a resolution issue of the ME method, we also use the BR method that shows better resolution for discrete structures in our mock data test to handle the same data. The results are shown in Fig. 7. And this time, to show exactly the effect of different default models, we plot the results with different default models separately in $\log$ scale. We also check the effect of including or excluding the data points of large $\tau$ since
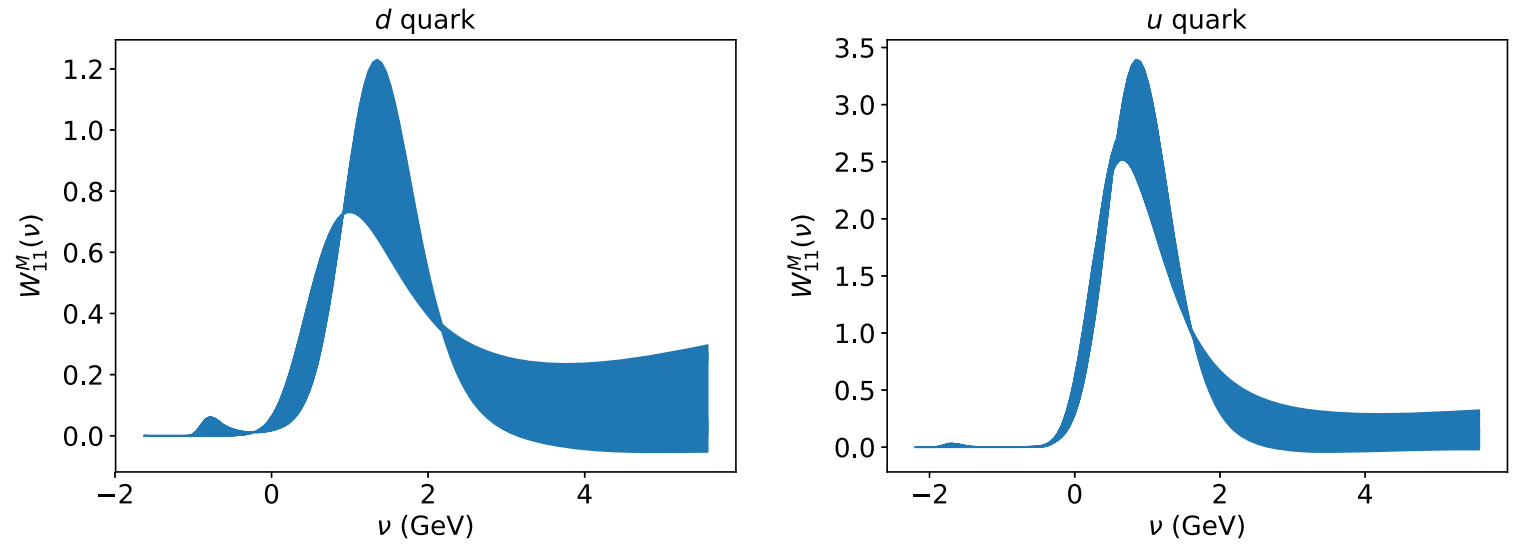

FIG. 6. The Minkowski hadronic tensor $W_{11}^{M}$ as a function of energy transfer $\nu$ reconstructed using the ME method for both $d$ and $u$ quarks. The error bands show the difference introduced by different ME parameters while no statistical errors are included. At some certain points, the error seems tiny which just indicates that different ME parameters result in similar results.
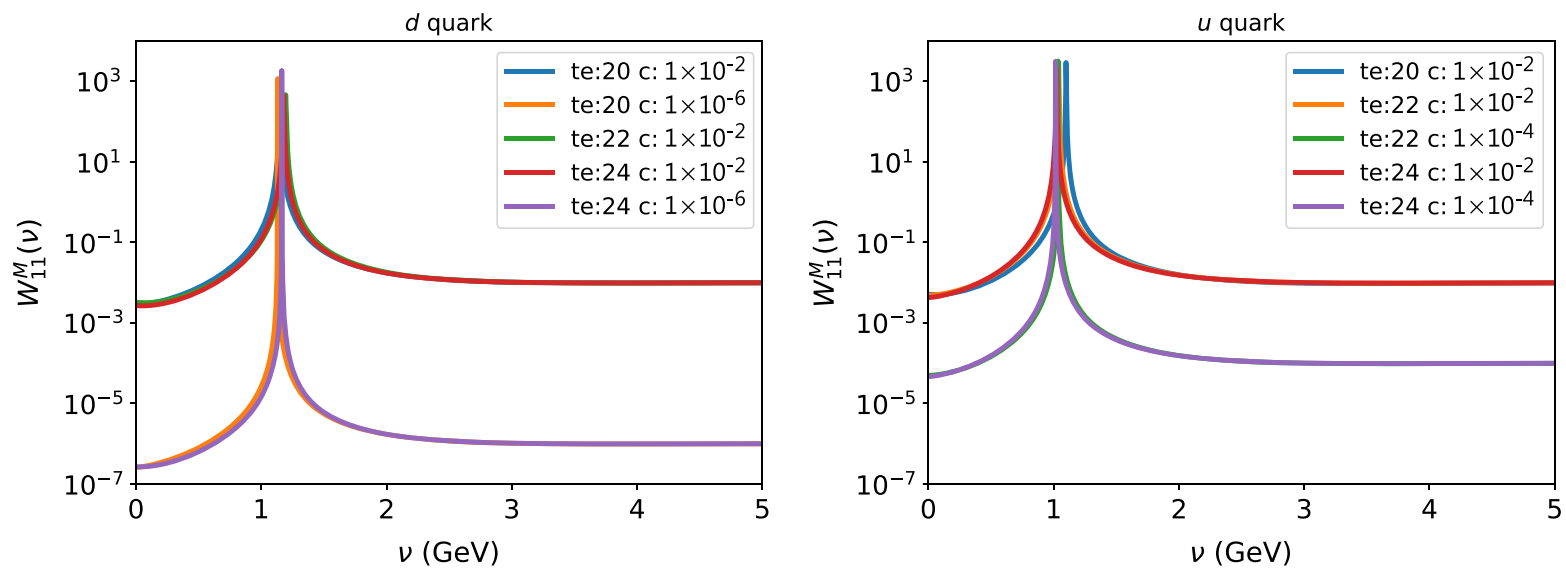

FIG. 7. The Minkowski hadronic tensor $W_{11}^{M}$ as a function of energy transfer $\nu$ reconstructed using the BR method for both $d$ and $u$ quarks. In the legend, te denotes the end point of $t_{2}$ we use in BR and $c$ is the value of the constant default model. After $\sim 2 \mathrm{GeV}$, the default models dominate the results. 
they can have large excited-state contaminations. This time, except that the peaks around $1 \mathrm{GeV}$ are much sharper than the ME case, the basic conclusion we learn is the same. No elastic contribution shows at $\nu=0$ and the only structure is around $1 \mathrm{GeV}$. When the energy transfer goes above, say, $2 \mathrm{GeV}$, the reconstructed results approach to the values of the default model which means the data have no constraint in that region.

From these two examples, we see that the elastic and the resonance contributions, where the energy transfer is not too large, can be extracted from the current setup. However, it seems that there is no contribution found in the DIS region. We will discuss the possible reason and solution in Sec. V.

It is suggested by Chambers et al. [7] that the structure functions can be extracted through time-ordered currentcurrent correlators on the lattice using the operator product expansion of the forward Compton amplitude. This is carried out by setting $\nu=0$ in the integral of Eq. (4) to avoid the divergence when $\nu-\left(E_{n}-E_{p}\right)>0$ as discussed in Sec. II after Eq. (5). However, this leads $W_{\mu \nu}^{\prime}(p, q, T)$ in Eq. (5) (considered to be the Compton amplitude $T_{\mu \nu}(p, q)$ in [7]) to

$$
\begin{aligned}
W_{\mu \nu}^{\prime}(T)= & \int d^{3} \vec{z} \frac{e^{i \vec{q} \cdot \vec{z}}}{4 \pi}\left[\left\langle p, s\left|J_{\mu}^{\dagger}(\vec{z})\right| 0\right\rangle\left\langle 0\left|J_{\nu}(0)\right| p, s\right\rangle T\right. \\
& \left.+\sum_{n \geq 1} \frac{e^{\left(-\left(E_{n}-E_{p}\right)\right) T}-1}{-\left(E_{n}-E_{p}\right)}\left\langle p, s\left|J_{\mu}^{\dagger}(\vec{z})\right| n\right\rangle\left\langle n\left|J_{\nu}(0)\right| p, s\right\rangle\right],
\end{aligned}
$$

where the state label 0 is for the nucleon state with momentum $\vec{p}+\vec{q}$ so that the first term accounts for the elastic scattering which diverges as $T$ and reflects the

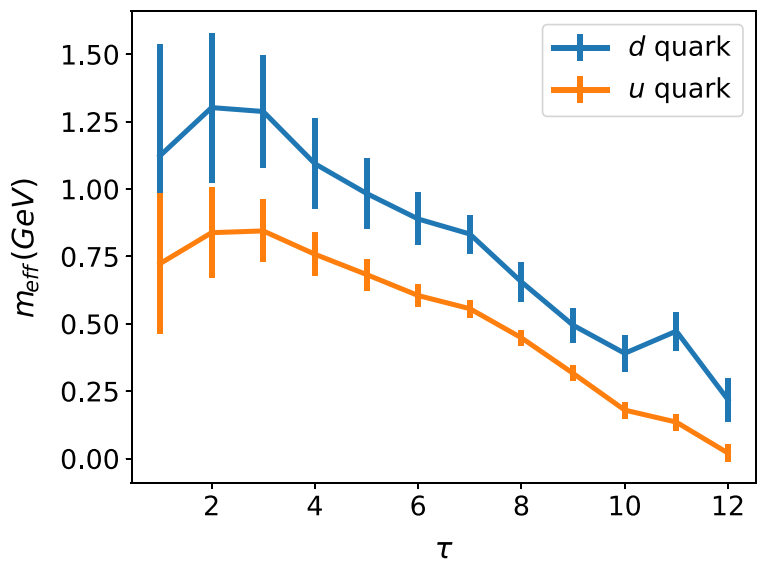

elastic scattering pole in Eq. (5). As $T \rightarrow \infty$, the contributions of the $n \geq 1$ states are suppressed by $1 / T$. However, as shown in Fig. 6, when $T$ is finite $(0.7 \mathrm{fm}$ in our case), the excited states including nucleon resonances and those in the SIS and DIS regions all contribute. When $Q^{2}$ is large (e.g., $12.7 \mathrm{GeV}^{2}$ in this case), the hadronic tensor for the elastic scattering is highly suppressed (by a factor of $\sim 10^{-5}$ ), whereas the resonance contribution around $1-2 \mathrm{GeV}$ at $Q^{2} \sim 9-11 \mathrm{GeV}^{2}$ is much larger as shown in Fig. 6.

To estimate how large a $\nu$ is needed for DIS, we can look at $W$, the total invariant mass of the hadronic final state

$$
W^{2}=(q+p)^{2}=m_{p}^{2}-Q^{2}+2 m_{p} \nu
$$

The global fittings of PDF usually take a cut with $W^{2}>10 \mathrm{GeV}^{2}$. When we take $Q^{2}=4 \mathrm{GeV}^{2}$, this gives $\nu>6.5 \mathrm{GeV}$. Therefore, taking $\nu=0$ in Eq. (4) will not yield PDF in the DIS region, which needs both $Q^{2}$ and $\nu$ to be large.

\section{DISCUSSION AND SUMMARY}

To explore the reason why there is no contribution for $\nu \gtrsim 2 \mathrm{GeV}$ in Figs. 6 and 7, we calculate the effective mass of the four-point functions which is a quick way to check the highest energy of intermediate states that our Euclidean hadronic tensor contains. The results are plotted in the left panel of Fig. 8. We see that for either the $u$ or $d$ quark, the highest effective mass is around $1 \mathrm{GeV}$, which means that there is simply no information of higher excitations which can be obtained for this particular case. This should be due to the lattice artifacts, since the lattice we are using has finite volume (resulting in discrete momenta and discrete

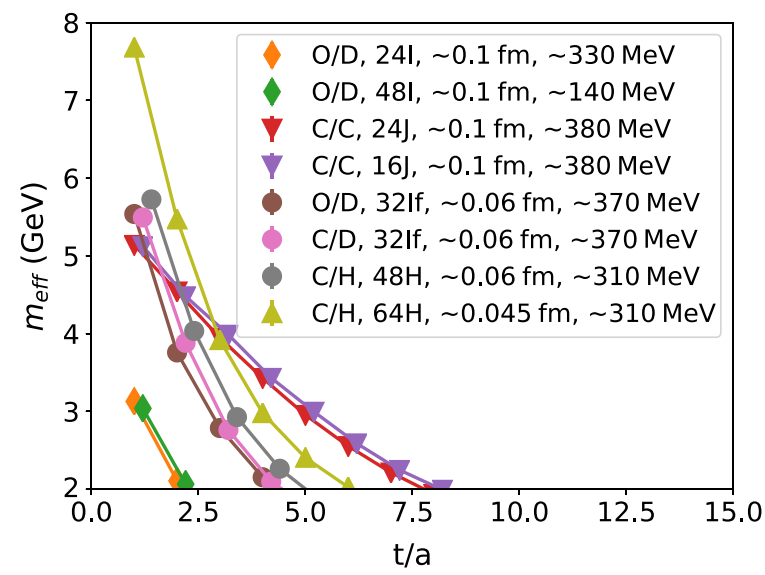

FIG. 8. The effective mass plot for the Euclidean hadronic tensor (left panel) and for the $\rho$ meson with different lattice setups (right panel), including different actions (overlap (O), clover (C), domain wall (D), and HISQ (H)), different spacial lattice spacings, and different pion masses. The information of the configurations used can be found in Refs. [29,31,32]. 24J and 16J are anisotropic lattices with $a_{s} / a_{t}=3.7$, so their highest effective masses are higher than those of 24I and 48I that have similar spacial lattice spacings. To increase visibility, some points in the right panel are shifted slightly in the horizontal direction. 
spectrum), finite lattice spacing (a UV cutoff), and unphysical pion mass (unphysical multiparticle states).

To sort out the most relevant lattice artifact, we calculate the effective mass of the $\rho$ meson with different lattice setups (the right panel of Fig. 8). The reason we choose to check the $\rho$ meson is because the hadronic tensor involves two vector currents inserted between the nucleon states and the correlator of $\rho$ can be treated as two vector currents inserted between the vacuum states. Although the exact value of how high we can reach in the $\rho$ meson case may not have much to do with the hadronic tensor case, how the lattice artifacts affect the effective mass should be relevant. The legend of the figure shows the features of the setups. Each label in the legend has four parts: valence quark type/ sea quark type ("O" denotes overlap, "D" domain wall, "C" clover, and "H" HISQ), spacial size plus a suffix which serves as an identifier, spacial lattice spacing, and sea pion mass. It is easy to see that, for 24I and 48I [29], although the pion masses and volumes are not the same, the highest effective masses are similar, around $3 \mathrm{GeV}$. For $24 \mathrm{~J}$ and $16 \mathrm{~J}$ [31], the spacial lattice spacings are similar to the ones of $24 \mathrm{I}$ and $48 \mathrm{I}(\sim 0.1 \mathrm{fm})$, but the highest effective masses can be higher than $5 \mathrm{GeV}$, which is because these two lattices are anisotropic and their temporal lattice spacings are about $0.035 \mathrm{fm}$. Then, for $48 \mathrm{H} \mathrm{[32]} \mathrm{and} \mathrm{the} \mathrm{two} \mathrm{setups} \mathrm{of} \mathrm{32If}$ [29], despite their different fermion actions and volumes, their highest effective masses are all about $5.5 \mathrm{GeV}$ and their lattice spacings are $\sim 0.06 \mathrm{fm}$. For the lattice with the

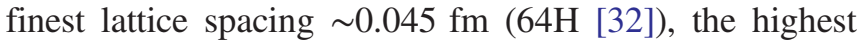
effective mass is close to $8 \mathrm{GeV}$. This test shows that the lattice spacing is the most important factor in order to have the information of higher excitations. In view of this comparison, the HISQ lattice with lattice spacing $\sim 0.045 \mathrm{fm}$ can be a better choice to reach $\nu>2 \mathrm{GeV}$ in our approach.

In this paper, we formulate our approach of calculating the hadronic tensor on the lattice. We point out that this is an approach that covers the inclusive contribution of all the intermediate states which is crucial to providing information for the neutrino scattering experiments at low energies. It is also promising to calculate the structure functions in the DIS region which can be used in the global fittings of parton distribution functions. However, solving the inverse problem is the most challenging part. We have implemented and tested three algorithms using mock data, showing that the BR method has the best resolution in extracting peak structures while BG and $\mathrm{ME}$ are more stable for the smooth Minkowski hadronic tensor at high energies. Realistic lattice results are presented for both the elastic case and a case with large momentum transfer. For the elastic case, the reconstructed Minkowski hadronic tensor from the ME method gives precisely the vector charge which shows the feasibility of this approach. For the latter case, the RES and possibly SIS contributions around $1 \mathrm{GeV}$ are observed but no information is obtained for higher excited states with $\nu>2 \mathrm{GeV}$.

A check of the effective masses of $\rho$ meson with different lattice setups indicates that, in order to reach higher energy transfers, using lattices with smaller lattice spacings is essential for this calculation. The HISQ lattice with lattice spacing $\sim 0.045 \mathrm{fm}$ should be suitable to study the neutrinonucleus scattering at DUNE where the beam energy is between $\sim 1$ and $\sim 7 \mathrm{GeV}$. In the future, working on lattices with lattice spacing of $0.03 \mathrm{fm}$ or even smaller would be desirable for studying the parton physics. Another possibility with the current $0.045 \mathrm{fm}$ lattice is to calculate the pion PDF. This might be an easier case, since we can have a better signal-to-noise ratio and we possibly do not need very large $Q^{2}$ or $\nu$ to enter the DIS region. The large mass gap between the pion and its first excited state will not be very problematic because, as long as the pion is boosted, the gap will become smaller and the multiparticle states will also contribute.

\section{ACKNOWLEDGMENTS}

K.-F. L. thanks X. Feng, A. Kronfeld, J.C. Peng, J. Qiu, and Y. Hatta for illuminating and useful discussions. We also thank the RBC and UKQCD Collaborations for providing their DWF gauge configurations. This work is supported in part by the U.S. DOE Grants No. DE-SC0013065 and No. DE-AC05-06OR23177 which is within the framework of the TMD Topical Collaboration. Y.-B. Y. is supported by Strategic Priority Research Program of Chinese Academy of Sciences, Grant No. XDC01040100. A. R. acknowledges support by the Research Council of Norway under the FRIPRO Young Research Talent Grants No. 286883 and No. 295310. This research used resources of the Oak Ridge Leadership Computing Facility at the Oak Ridge National Laboratory, which is supported by the Office of Science of the U.S. Department of Energy under Contract No. DEAC05-00OR22725. This work used Stampede time under the Extreme Science and Engineering Discovery Environment, which is supported by National Science Foundation Grant No. ACI-1053575. We also thank the National Energy Research Scientific Computing Center for providing high performance computing resources that have contributed to the research results reported within this paper. We acknowledge the facilities of the USQCD Collaboration used for this research in part, which are funded by the Office of Science of the U.S. Department of Energy. 
[1] K.-F. Liu and S.-J. Dong, Phys. Rev. Lett. 72, 1790 (1994).

[2] K.-F. Liu, Phys. Rev. D 62, 074501 (2000).

[3] K.-F. Liu, Proc. Sci., LATTICE2015 (2016) 115 [arXiv: 1603.07352].

[4] J. Liang, K.-F. Liu, and Y.-B. Yang, EPJ Web Conf. 175, 14014 (2018).

[5] X. Ji, Phys. Rev. Lett. 110, 262002 (2013).

[6] H.-W. Lin, J.-W. Chen, X. Ji, L. Jin, R. Li, Y.-S. Liu, Y.-B. Yang, J.-H. Zhang, and Y. Zhao, Phys. Rev. Lett. 121, 242003 (2018).

[7] A. J. Chambers, R. Horsley, Y. Nakamura, H. Perlt, P. E. L. Rakow, G. Schierholz, A. Schiller, K. Somfleth, R. D. Young, and J. M. Zanotti, Phys. Rev. Lett. 118, 242001 (2017).

[8] A. V. Radyushkin, Phys. Rev. D 96, 034025 (2017).

[9] K. Orginos, A. Radyushkin, J. Karpie, and S. Zafeiropoulos, Phys. Rev. D 96, 094503 (2017).

[10] Y.-Q. Ma and J.-W. Qiu, Phys. Rev. Lett. 120, 022003 (2018).

[11] R. S. Sufian, J. Karpie, C. Egerer, K. Orginos, J.-W. Qiu, and D. G. Richards, Phys. Rev. D 99, 074507 (2019).

[12] K.-F. Liu, W.-C. Chang, H.-Y. Cheng, and J.-C. Peng, Phys. Rev. Lett. 109, 252002 (2012).

[13] J. Liang, M. Sun, Y.-B. Yang, T. Draper, and K.-F. Liu, arXiv:1901.07526.

[14] R. Acciarri et al. (DUNE Collaboration), arXiv:1512.06148.

[15] L. Alvarez-Ruso, M. S. Athar, M. Barbaro, D. Cherdack, M. Christy, P. Coloma, T. Donnelly, S. Dytman, A. de Gouvêa, R. Hill et al., Prog. Part. Nucl. Phys. 100, 1 (2018).

[16] J. A. Formaggio and G. P. Zeller, Rev. Mod. Phys. 84, 1307 (2012).
[17] A. S. Kronfeld, D. G. Richards, W. Detmold, R. Gupta, H.-W. Lin, K.-F. Liu, A. S. Meyer, R. Sufian, and S. Syritsin (USQCD Collaboration), Eur. Phys. J. A 55, 196 (2019).

[18] G. Backus and F. Gilbert, Geophys. J. Int. 16, 169 (1968).

[19] G. Backus and F. Gilbert, Phil. Trans. R. Soc. A 266, 123 (1970).

[20] M. T. Hansen, H. B. Meyer, and D. Robaina, Phys. Rev. D 96, 094513 (2017).

[21] S. Kim, P. Petreczky, and A. Rothkopf, J. High Energy Phys. 11 (2018) 088.

[22] E. Rietsch, J. Geophys. 42, 489 (1977).

[23] M. Asakawa, T. Hatsuda, and Y. Nakahara, Prog. Part. Nucl. Phys. 46, 459 (2001).

[24] R. K. Bryan, Eur. Biophys. J. 18, 165 (1990).

[25] A. Rothkopf, J. Comput. Phys. 238, 106 (2013).

[26] Y. Burnier and A. Rothkopf, Phys. Rev. Lett. 111, 182003 (2013).

[27] C. S. Fischer, J. M. Pawlowski, A. Rothkopf, and C. A. Welzbacher, Phys. Rev. D 98, 014009 (2018).

[28] J. Karpie, K. Orginos, A. Rothkopf, and S. Zafeiropoulos, J. High Energy Phys. 04 (2019) 057.

[29] T. Blum et al. (RBC and UKQCD Collaborations), Phys. Rev. D 93, 074505 (2016).

[30] T.-P. Cheng and L.-F. Li, Gauge Theory of Elementary Particle Physics, Oxford Science Publications (Clarendon Press, Oxford University Press, New York, 1984).

[31] H.-W. Lin et al. (Hadron Spectrum Collaboration), Phys. Rev. D 79, 034502 (2009).

[32] A. Bazavov et al. (MILC Collaboration), Rev. Mod. Phys. 82, 1349 (2010). 